\title{
Rheumatic mitral stenosis in association with partial anomalous pulmonary venous return
}

\author{
J. M. REID, R. S. BARCLAY, J. G. STEVENSON, \\ T. M. WELSH, AND N. MCSW AN \\ From the Cardio-Thoracic Unit, Mearnskirk Hospital, Glasgow
}

\begin{abstract}
An unusual case is presented, in which mitral stenosis was accompanied by a persistent left superior vena cava communicating through a venous channel with the left pulmonary veins. The pulmonary veins were normally connected to the left atrium, but, due to elevation of the left atrial pressure from the mitral valve lesion, pulmonary venous blood was directed up the left superior vena cava to reach the right atrium. Although from an anatomical viewpoint this did not constitute true anomalous pulmonary venous drainage, the condition behaved as such haemodynamically. It was only at operation that the diagnosis was firmly established, but after mitral valvotomy the abnormal venous channel was obliterated by simple ligation without incident.
\end{abstract}

Total anomalous pulmonary venous drainage customarily produces severe cardiac disability either in early infancy (Hastreiter, Paul, Molthan, and Miller, 1962) or in later life, depending on the presence or otherwise of an atrial septal defect and also on its size. Conversely, patients with partial anomalous venous drainage may remain symptomfree either till adult life or indefinitely, depending on whether there is an accompanying malformation and on the type of abnormality of venous return. Blake, Hall, and Manion (1965) discussed 27 different varieties of this condition.

We wish to record an unusual case in which a left superior vena cava communicated with the left pulmonary veins which were also normally connected to the left atrium. But for the supervention of mitral stenosis, the condition could have remained dormant, perhaps for the remainder of the patient's life. Persisting left anterior cardinal vein, left vertical vein, and left superior vena cava are all synonymous terms, but the last is least cumbersome and certainly more descriptive.

\section{CASE HISTORY}

The patient, a man aged 33 years, first learned that he had a heart murmur when he was invalided from the Army in 1956 at the age of 23 years with the diagnosis of mitral valve disease. In the same year he underwent surgica! treatment for haemorrhoids but thereafter remained well until 1962, when an emergency appendicectomy was performed. It was not until early 1965 that he developed any cardiac symptoms, which initially took the form of grade I exertional dyspnoea not progressive in type. In April of the following year (1966) he had a small but brisk haemoptysis, which recurred later in the same year and led to his admission to hospital for further investigation.

Clinical examination revealed a relatively fit young man with no evidence of cyanosis or finger clubbing. Blood pressure measured $120 / 80 \mathrm{~mm}$. $\mathrm{Hg}$, and the heart was in sinus rhythm. Right ventricular pulsation was present, but no thrills were palpable. There was a grade II systolic murmur down the left sternal border, maximal at the second and third interspaces, and the second pulmonary sound was accentuated. Although the first sound was loud, no diastolic murmur was audible at the mitral area. The electrocardiogram showed bifid $\mathbf{P}$ waves indicative of left atrial enlargement, right axis deviation, and right ventricular hypertrophy. Cardiac radiographs and screening demonstrated pulmonary congestion and enlargement of the heart shadow, affecting principally the left atrium and right ventricle, with horizontal lines at both lung bases. Sputum examination was negative for tubercle bacilli, and blood urea was $30 \mathrm{mg}$. per $100 \mathrm{ml}$. Haemoglobin was $97 \%$, P.C.V. $41 \%$, M.C.H.C. $34.5 \%$, white cell count 7,700/c.mm., and E.S.R. $2 \mathrm{~mm}$. in the first hour. Urine examination was also negative.

Cardiac catheterization was performed, with the results shown in the Table.

A rise in oxygen saturation was observed between high and low superior vena cava, this being maintained to the lung periphery. This suggested to us a large intracardiac shunt at the level of the superior vena cava due to either a sinus venosus atrial septal defect or anomalous pulmonary venous drainage Despite repeated attempts, the catheter could not be 
T A B L E

RESULTS OF CARDIAC CATHETERIZATION

\begin{tabular}{|c|c|c|c|c|}
\hline & Site & & $\begin{array}{c}\text { Dynamic } \\
\text { Pressure }(\mathrm{mm} . \mathrm{Hg})\end{array}$ & $\begin{array}{l}\text { \% Oxygen } \\
\text { Saturation }\end{array}$ \\
\hline S.V.C. & .. & .. & - & 70 \\
\hline (low) & $\ldots$ & .. & - & 93 \\
\hline I.V.C. & . & . & - & 73 \\
\hline R.A. & .. & .. & $5 / 0$ & 94 \\
\hline R.V. & $\because$ & $\because$ & $50 / 0$ & 92 \\
\hline M.P.A. & $\ldots$ & $\therefore$ & $50 / 30$ & 90 \\
\hline Wedge & $\therefore$ & $\therefore$ & $10 / 2$ & 95 \\
\hline Femoral arte & tery & $\therefore$ & $130 / 70$ & 99 \\
\hline
\end{tabular}

made to enter the left atrium or any of the pulmonary veins. Dye dilution curves performed in both the superior vena cava and the main pulmonary artery demonstrated a large left-to-right shunt; but in the right pulmonary artery a normal dye curve was obtained. These findings were considered to be consistent with a diagnosis of anomalous pulmonary venous drainage from the left lung. Angiocardiography had to be abandoned due to the onset of a serious cardiac arrhythmia following the introduction of a National Institute of Health catheter.

It was decided to proceed with the operation using cardiopulmonary bypass, and this was performed on 15 March 1967. A bilateral thoracotomy was done: the heart was examined externally and this, combined with digital exploration of both atria, revealed the true situation (see Figure). A vertical venous channel linked the left pulmonary veins extrapericardially with the left innominate vein. This in turn drained into an

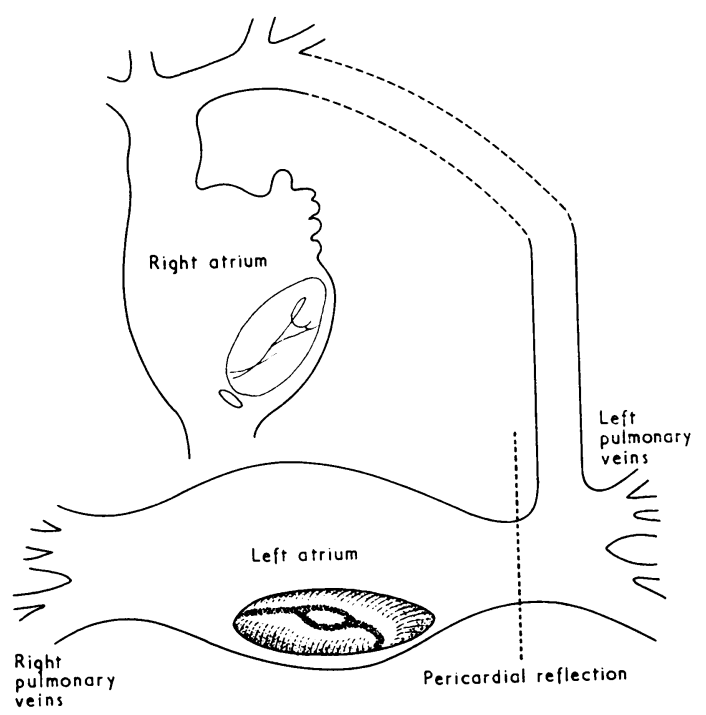

FIGURE Rheumatic mitral stenosis associated with partial anomalous pulmonary venous return. The diagram illustrates the findings at operation. enlarged superior vena cava and thence to the right $\stackrel{\overrightarrow{\vec{S}}}{\stackrel{\vec{P}}{+}}$ atrium. The pulmonary veins from both lungs were connected normally to the left atrium, and the atrial septum was intact. There was extreme stenosis of the $\frac{\bar{\rho}}{\vec{\alpha}}$ mitral valve, the orifice measuring no more than $1 \mathrm{~cm} . \widehat{\Phi}$ Initial occlusion of the venous channel linking the left pulmonary veins to the superior vena cava pro- $\omega$ duced an abrupt elevation in pressure in both the $\overrightarrow{0}$ right and left pulmonary veins. It was then decided to proceed with mitral valvotomy, following which $\vec{\omega}$

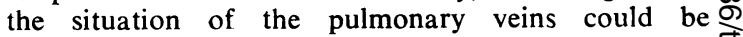
reassessed. Using the Tubb's dilator, the valve was $\vec{\sim}$ dilated to $3.0 \mathrm{~cm}$.; the valve remained competent and its size was considered adequate in relation to the $\omega$ underdeveloped dimensions of the mitral annulus and $\stackrel{+}{-}$ the left ventricle. Repeat clamping of the abnormal $\overrightarrow{0}$ vein did not show a rise in pressure in either the left or right pulmonary veins, and it was ligated. Cardio- $\frac{}{\square}$ pulmonary bypass was not required for these manœuvres.

The patient made a smooth recovery from his operation, although atrial fibrillation developed on $\stackrel{\circ}{\mathcal{T}}$ the third post-operative day. Sinus rhythm was $\vec{\varphi}$ restored by cardioversion.

\section{DISCUSSION}

Anomalous return of the pulmonary veins can be a comparatively simple condition or a highly complex abnormality. Blake et al. (1965) docu- $\cong$ mented 113 patients with anomalous pulmonary $\overrightarrow{\overrightarrow{0}}$ venous drainage, and they described 27 variations. Referring to abnormal drainage of pulmonary veins into a persistent left superior vena cava. Snellen and Dekker (1963) reported 25 personal cases. They divided their patients into three categories, each with four subgroups, depending $x$ on whether the drainage was partial or total, and whether persistence of the left superior vena cava was complete or incomplete. Our case might fit into group C, subgroup CIII of this classification, in which there is partial persistence of the left 음 superior vena cava, and one or more pulmonary $\rightarrow$ veins are normally connected to the left atrium. Zion (1956) recorded a case of mitral stenosis $N$ occurring in a woman aged 42 years in whom there was also partial anomalous pulmonary 0 venous drainage into a left superior vena cava. The condition was recognized only at operation carried out as an emergency because of theo patient's grave cardiac state. It was observed that $\bar{\Phi}$ the left superior pulmonary vein entered a left $\stackrel{\oplus}{+}$ superior vena cava which in turn drained to the right atrium. The left inferior pulmonary vein was normally connected with the left atrium. $\stackrel{\mathbb{Q}}{\overparen{Q}}$ Mitral valvotomy was performed, no attempt $\stackrel{\mathbb{Q}}{\varrho}$ being made to deal with the other abnormality $\sigma$ present. The patient derived considerable benefit 
from the operation. Aldridge and Wigle (1965), reviewing a total of 12 previous cases of mitral stenosis (five of whom were rheumatic in origin and seven congenital) associated with partial anomalous pulmonary venous drainage and intact septum, added a further instance. This occurred in a 22-year-old woman admitted in severe congestive cardiac failure, who died before operation could be performed. At necropsy the presence of congenital mitral stenosis was confirmed, associated with drainage of the pulmonary veins from the right upper and middle lobes to the right atrium.

The particular abnormality found in our patient in association with mitral stenosis has not hitherto been reported. The pulmonary veins from both right and left lungs drained normally to the left atrium, but the left veins also communicated through a venous channel to a left superior vena cava. Anatomically the condition was thus not true anomalous pulmonary venous drainage but was potentially so from a haemodynamic point of view. By itself this was comparatively innocuous and would have remained so had the patient not developed rheumatic mitral stenosis. The consequent rise in left atrial pressure would thus produce an impediment to venous return from the left lung, which in the circumstances would then traverse the easier and less pressurized channel up the left superior vena cava into the right atrium. That this return through the left superior vena cava was substantial is manifest from the high oxygen saturation obtained in the right atrium at catheterization and reflected in the pulmonary systemic ratio of $2 \cdot 6: 1$. At operation initial clamping of the abnormal venous channel caused a marked elevation in pulmonary venous pressure, but following surgical relief of the mitral obstruction this rise did not occur, and it was then considered feasible to proceed with ligation of this channel.

The authors are indebted to Dr. A. J. V. Cameron, Western Infirmary, Glasgow, under whose care the patient was admitted for investigation and who subsequently referred him for operation.

\section{REFERENCES}

Aldridge, H. E., and Wigle, E. D. (1965). Partial anomalous venous drainage with intact interatrial septum associated with congenital mitral stenosis. Circulation, 31, 579 .

Blake, H. A., Hall, R. J., and Manion, W. C. (1965). Anomalous pulmonary venous return. Ibid., 32, 406.

Hastreiter, A. R., Paul, M. H., Molthan, M. E., and Miller, R. A. (1962). Total anomalous pulmonary venous connection with severe pulmonary venous obstruction: a clinical entity. Ibid., 25, 916 .

Snellen, H. A., and Dekker, A. (1963). Anomalous pulmonary venous drainage in relation to left superior vena cava and coronary sinus. Amer. Heart J., 66, 184.

Zion, M. M. (1956). Mitral stenosis associated with anomalous pulmonary venous drainage into a left superior vena cava. Brit. med. J., 1, 1020. 